\title{
The role of immunotherapy in the management of patients with renal cell carcinoma and brain metastases
}

\author{
Jaleh Fallah ${ }^{1}$, Manmeet S. Ahluwalia ${ }^{1,2}$ \\ ${ }^{1}$ Taussig Cancer Institute, ${ }^{2}$ Burkhardt Brain Tumor and Neuro-Oncology Center, Cleveland Clinic, Cleveland Ohio, OH, USA \\ Correspondence to: Manmeet S. Ahluwalia, MD, FACP. Professor, Department of Medicine, Burkhardt Brain Tumor and Neuro-Oncology Center, \\ Neurological Institute, Cleveland Clinic, 9500 Euclid Ave., S73, Cleveland, OH 44195, USA. Email: ahluwam@ccf.org. \\ Provenance: This is an invited article commissioned by the Section Editor Dr. Xiao Li (Department of Urology, Jiangsu Cancer Hospital, Jiangsu \\ Institute of Cancer Research, Nanjing Medical University Affiliated Cancer Hospital, Nanjing, China). \\ Comment on: Flippot R, Dalban C, Laguerre B, et al. Safety and Efficacy of Nivolumab in Brain Metastases From Renal Cell Carcinoma: Results of \\ the GETUG-AFU 26 NIVOREN Multicenter Phase II Study. J Clin Oncol 2019;37:2008-16.
}

Submitted Sep 22, 2019. Accepted for publication Sep 30, 2019.

doi: $10.21037 /$ atm.2019.10.31

View this article at: http://dx.doi.org/10.21037/atm.2019.10.31

Patients with metastatic renal cell carcinoma (mRCC) who have metastases to the brain have poor prognosis and median overall survival (OS) of 6 to 10 months $(1,2)$. Local treatments such as surgical resection, stereotactic radiosurgery (SRS) and whole brain radiation therapy (WBRT) are the mainstay of treatment in these patients. The decision to choose one of these approaches depends on the number, size and location of the brain lesions, presence or absence of symptoms and the level of expertise available in the treating facility. Surgical resection is generally performed in patients with a solitary and easily accessible brain lesion. WBRT is mostly utilized in the treatment of patients with numerous brain metastases (mostly for $>10$ lesions) and SRS is the treatment of choice in patients with limited brain metastases (up to 10 lesions). The rate of local control with SRS is more than $90 \%(3,4)$ and overall, it has been found to be safe and well-tolerated by patients.

Recent clinical trials in patients with melanoma have shown efficacy of checkpoint inhibitors in the treatment of brain metastases. In phase II clinical trials of pembrolizumab or ipilimumab in patients with melanoma with asymptomatic previously untreated melanoma, the objective response rate (ORR) was 22-26\% (Table 1). Combination of ipilimumab plus nivolumab was studied in two phase II clinical trials in patients with melanoma with asymptomatic previously untreated brain metastases and resulted in $46-57 \%$ intracranial response, including $17-26 \%$ intracranial complete response (5-9).

Using checkpoint inhibitors as monotherapy or in combination with chemotherapy in patients with metastatic non-small cell lung cancer (NSCLC) has shown efficacy in management of brain metastases. In a phase II clinical trial, 18 patients with NSCLC with asymptomatic brain metastases previously untreated or progressing on local treatment were treated with pembrolizumab alone and had intracranial response rate $33 \%$ (9). Most of the patients with NSCLC with brain metastases who responded to treatment had a durable response (13). KEYNOTE-189 is a randomized double-blinded phase III clinical trial of pembrolizumab plus chemotherapy versus chemotherapy alone in patients with metastatic non-squamous NSCLC. Among 606 patients enrolled into this clinical trial, 108 patients had brain metastases. The results of the study showed that patients with NSCLC with brain metastases who received combination of pembrolizumab plus chemotherapy has significantly better survival outcomes compared to those who were treated with chemotherapy only, hazard ratio (HR) for OS 0.36 , HR for progressionfree survival (PFS) 0.42 (10).

Emergence of vascular endothelial growth factor (VEGF) inhibitors and checkpoint inhibitors has revolutionized the treatment of mRCC. Phase III clinical trials of checkpoint inhibitors in patients with $\mathrm{mRCC}$ resulted in durable responses and improved survival outcomes in frontline and second line settings. CheckMate-025 clinical trial demonstrated significant improvement in ORR and OS in patients with mRCC treated with nivolumab compared with everolimus, after progression on a previous anti- 
Table 1 The efficacy of immunotherapy in management of brain metastases

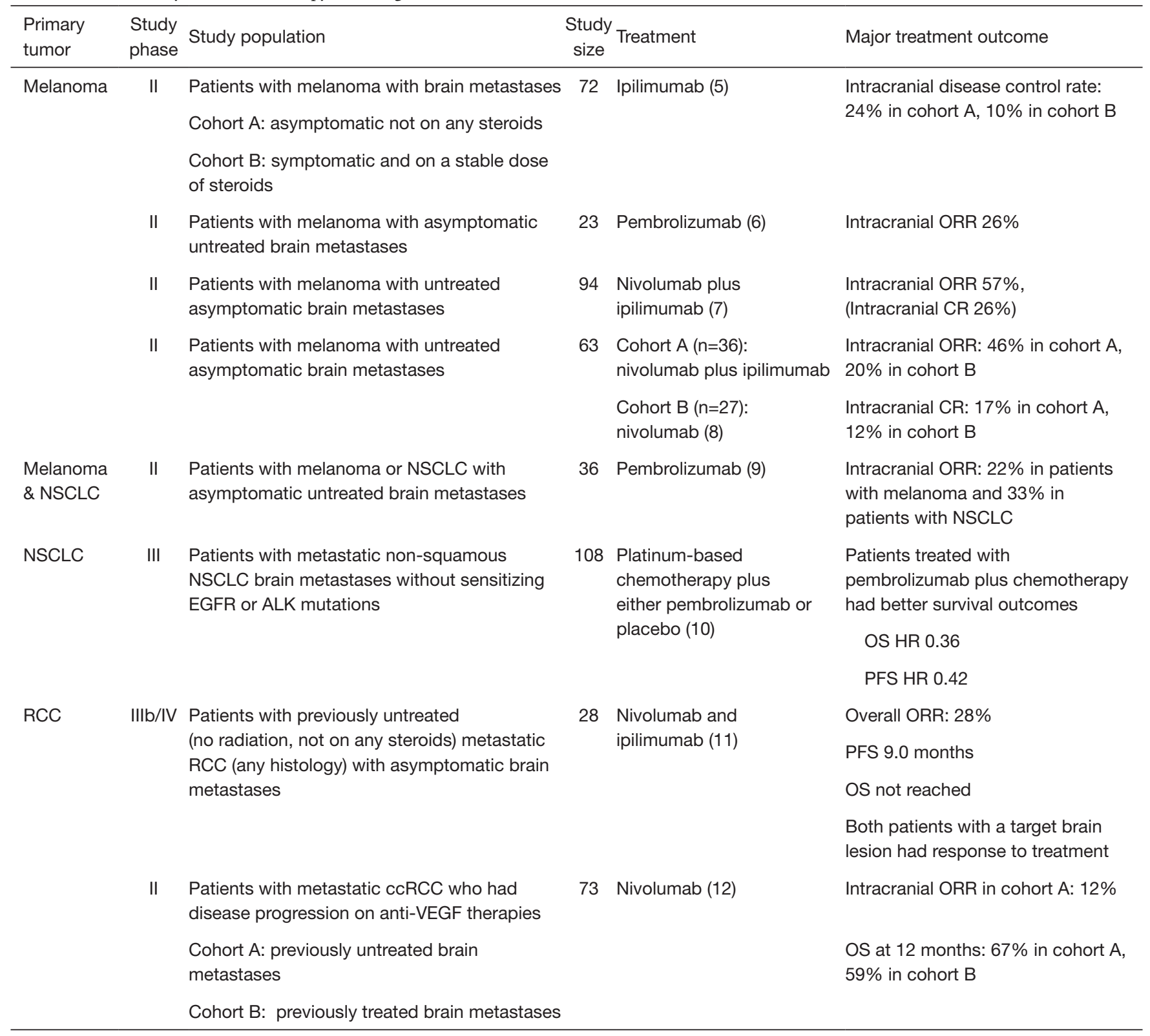

RCC, renal cell carcinoma; NSCLC, non-small cell lung cancer; OS, overall survival; PFS, progression-free survival; ORR objective response rate.

VEGF treatment (14). The CheckMate-214 study showed significantly better ORR and longer OS with ipilimumab plus nivolumab followed by maintenance nivolumab compared to sunitinib (OS HR 0.63) (15).

The combination of checkpoint inhibitors and VEGFTKIs were studied in two phase III clinical trials for first line treatment of mRCC. In KEYNOTE-426, patients with previously untreated $\mathrm{mRCC}$ who received the combination of pembrolizumab plus axitinib had significantly better survival outcomes compared to those who were treated with sunitinib [HR for OS $0.53,95 \%$ confidence interval (CI): 0.38-0.74] (16). In JAVELIN Renal 101, the combination of avelumab plus axitinib resulted in significantly better PFS compared to sunitinib in patients with previously untreated mRCC (HR for PFS 0.69, 95\% CI: 0.56-0.84) (17). Based on the results of these clinical trials, the combination of ipilimumab plus nivolumab, pembrolizumab plus axitinib, and avelumab plus axitinib have been approved by the Food 
and Drug Administration (FDA) for treatment of mRCC in the frontline setting. However, most of the pivotal clinical trials in patients with mRCC, excluded patients with brain metastases and those which included these patients did not report the outcomes in this subgroup of patients.

Two recent clinical trials have studied the safety and efficacy of checkpoint inhibitors in patients with $\mathrm{mRCC}$ with brain metastases and showed that the treatment with nivolumab as monotherapy or in combination with ipilimumab in patients with mRCC brain metastases is safe $(11,12)$.

CheckMate-920 is a phase IIIb/VI clinical trial to study the safety and efficacy of the combination of ipilimumab plus nivolumab followed by maintenance nivolumab in previously untreated mRCC. The cohort 3 of this clinical trial enrolled patients with $\mathrm{mRCC}$ of any histology who had asymptomatic brain metastases. Among 28 patients who were enrolled in the brain metastases cohort, 21 patients had non-target (smaller than $10 \mathrm{~mm}$ or previously irradiated) brain lesions. The minimum follow up at the time of interim analysis was 6.5 months at which point, 2 patients had significant decrease in size of the target brain lesion and 5 patients (17\%) had progression of non-target brain lesions or developed new brain metastases (11).

The GETUG-AFU 26 NIVOREN Brain Metastases study is a phase II clinical trial which enrolled patients with mRCC brain metastases who had disease progression on at least one prior anti-VEGF therapy. Patients with asymptomatic previously untreated target brain lesions were included in cohort A (39 patients) and those with previously treated target brain metastases were included in cohort B (34 patients). In Nivoren-26 clinical trial, the ORR was $19 \%$ which was lower than the ORR 25\% in CheckMate-025. The worse outcomes in Nivoren-26 can be due to the previously heavily treated patient population which are less likely to respond to treatment. In Nivoren-26 study, 31\% of the patients had 2 prior anti-VEGF therapies and $31 \%$ of the patients were previously treated with at least 3 antiVEGF therapy, whereas in CheckMate-025 study, 72\% of the patients had only one prior anti-VEGF therapy. Moreover, RCC cells which invade into the brain may be less responsive to immunotherapy, which might have led to worse outcomes in patients in the Nivoren-26 study compared to CheckMate-025 study which excluded patients with brain metastases $(12,14)$.

In the GETUG-AFU 26 NIVOREN Brain Metastases study, the intracranial ORR in cohort A (primary endpoint) was $12 \%$ which was only seen in patients with a solitary brain lesion smaller than $10 \mathrm{~mm}$. The intracranial median PFS in cohort A and cohort B were 2.7 and 4.8 months, respectively. The 12 -month OS was $67 \%$ in cohort $\mathrm{A}$ and $59 \%$ in cohort B. More than half of the patients $(51 \%)$ in cohort A needed treatment with corticosteroids compared to $27 \%$ of the patients in cohort B. Subsequent local therapy (surgical resection or radiation therapy) was needed in $72 \%$ of the patients in cohort $\mathrm{A}$ and $21 \%$ of the patients in cohort B (12).

Checkpoint inhibitors treat brain metastases by activating the peripheral T-cells which circulate in the blood and access the brain metastases. However, systemic treatment with checkpoint inhibitors is not adequate to control the intracranial metastatic disease. Previous studies of anti-VEGF therapy and checkpoint inhibitors in patients with mRCC brain metastases showed improved OS despite lack of local control which suggests that the survival benefit with systemic therapy was mainly due to control of the extracranial disease $(18,19)$.

Using concurrent immunotherapy with radiation therapy can yield a synergistic effect against tumor cells. One of the mechanisms that malignant cells evade the immune system is by decreasing the expression of the tumor antigens. Radiation therapy to solid tumors results in necrosis of the tumor tissue and release of tumor antigens into the blood which increases the exposure of the immune system into the tumor antigens. PEMBRO$\mathrm{RT}$ is a phase II clinical trial which randomized 72 patients with metastatic NSCLC to receive the combination of pembrolizumab plus SBRT or pembrolizumab only. No increase in toxicity was observed in the combination arm. The ORR at 12 weeks was significantly better in patients treated with combination therapy (36\%) compared with those treated with pembrolizumab monotherapy (18\%). However, there was no statistically significant difference in PFS and OS between the treatment arms. A subgroup analysis showed that the clinical benefit from addition of RT to pembrolizumab was mainly seen among patients with negative PD-L1 expression (20). Several clinical trials are currently investigating the abscopal effect with combination of RT and immunotherapy in patients with Hodgkin's lymphoma (NCT03480334), melanoma (NCT03354962), esophageal and gastric cancer (NCT02830594), and carcinoma of unknown primary (NCT03396471).

Retrospective studies suggest a synergy between SRS of brain lesions and systemic treatment with checkpoint inhibitors. In a retrospective study of 260 patients with melanoma, NSCLC and RCC with brain 
metastases, patients treated with stereotactic radiosurgerystereotactic radiation therapy (SRS-SRT) and concurrent immunotherapy (within 2 weeks of radiation) had significantly longer OS compared to those who were treated with SRS-SRT only (HR 2.69, P 0.002) or non-concurrent immunotherapy (HR 2.40, P 0.006). Moreover, there was a significant decrease in incidence of new brain metastases in patients treated with concurrent SRS plus checkpoint inhibitors (odds ratio 0.33, $\mathrm{P}=0.045$ ) (21). Several ongoing clinical trials are investigating the safety and efficacy of the combination of SRS plus checkpoint inhibitors in patients with melanoma and NSCLC with brain metastases (NCT03340129, NCT02858869).

Delay in local control of brain lesions is another limitation of systemic treatment for management of brain metastases. In patients with mRCC, the median time to response to checkpoint inhibitor or anti-VEGF therapy is approximately 3 months (14-17). Therefore, in patients with brain metastases, particularly those with symptomatic and active metastatic lesions, rapid control of brain metastases by traditional approaches such as SRS or surgery is necessary.

For management of patients with mRCC and brain metastases, we recommend a multi-modality approach, using a combination of local therapies such as SRS to achieve local control of the intracranial disease, plus systemic treatment to control the intracranial and extracranial disease. Use of local treatments such as SRS in treatment of mRCC is particularly needed in patients with multiple, large or symptomatic brain lesions. Future studies need to assess the intracranial efficacy of the combination of anti-VEGF therapy plus immunotherapy in patients with mRCC, as well as the safety and efficacy of the combination of the systemic treatments with radiation therapy in these patients, and the interval and sequence of local and systemic treatments for management of patients with RCC and brain metastases.

\section{Acknowledgments}

None.

\section{Footnote}

Conflicts of Interest: MS Ahluwalia: Receipt of grants/ research supports: Astrazeneca, Abbvie, BMS, Bayer, Incyte, Pharmacyclics, Novocure, Merck; Receipt of honoraria or consultation fees: Elsevier, Wiley, Astrazeneca, Abvvie, VBI Vaccines, Flatiron, Varian Medical Systems,
Prime Education, Bayer, karyopharm, Tocagen, Forma therapeutics; Stock shareholder: Doctible, Mimivax. The other author has no conflicts of interest to declare.

Ethical Statement: The authors are accountable for all aspects of the work in ensuring that questions related to the accuracy or integrity of any part of the work are appropriately investigated and resolved.

\section{References}

1. Du Y, Pahernik S, Hadaschik B, et al. Impact of resection and systemic therapy on the survival of patients with brain metastasis of metastatic renal cell carcinoma. J Neurooncol 2016;130:221-8.

2. Sun M, De Velasco G, Brastianos PK, et al. The Development of Brain Metastases in Patients with Renal Cell Carcinoma: Epidemiologic Trends, Survival, and Clinical Risk Factors Using a Population-based Cohort. Eur Urol Focus 2019;5:474-81.

3. Wowra B, Siebels M, Muacevic A, et al. Repeated gamma knife surgery for multiple brain metastases from renal cell carcinoma. J Neurosurg 2002;97:785-93.

4. Sheehan JP, Sun MH, Kondziolka D, et al. Radiosurgery in patients with renal cell carcinoma metastasis to the brain: long-term outcomes and prognostic factors influencing survival and local tumor control. J Neurosurg 2003;98:342-9.

5. Margolin K, Ernstoff MS, Hamid O, et al. Ipilimumab in patients with melanoma and brain metastases: an openlabel, phase 2 trial. Lancet Oncol 2012;13:459-65.

6. Kluger HM, Chiang V, Mahajan A, et al. Long-Term Survival of Patients With Melanoma With Active Brain Metastases Treated With Pembrolizumab on a Phase II Trial. J Clin Oncol 2019;37:52-60.

7. Tawbi HA, Forsyth PA, Algazi A, et al. Combined Nivolumab and Ipilimumab in Melanoma Metastatic to the Brain. N Engl J Med 2018;379:722-30.

8. Long GV, Atkinson V, Lo S, et al. Combination nivolumab and ipilimumab or nivolumab alone in melanoma brain metastases: a multicentre randomised phase 2 study. Lancet Oncol 2018;19:672-81.

9. Goldberg SB, Gettinger SN, Mahajan A, et al. Pembrolizumab for patients with melanoma or non-smallcell lung cancer and untreated brain metastases: early analysis of a non-randomised, open-label, phase 2 trial. Lancet Oncol 2016;17:976-83.

10. Gandhi L, Rodriguez-Abreu D, Gadgeel S, et al. 
Pembrolizumab plus Chemotherapy in Metastatic NonSmall-Cell Lung Cancer. N Engl J Med 2018;378:2078-92.

11. Emamekhoo H, Olsen M, Carthon BC, et al. Safety and efficacy of nivolumab plus ipilimumab (NIVO+IPI) in patients with advanced renal cell carcinoma (aRCC) with brain metastases: Interim analysis of CheckMate 920. J Clin Oncol 2019;37:abstr 4517.

12. Flippot R, Dalban C, Laguerre B, et al. Safety and Efficacy of Nivolumab in Brain Metastases From Renal Cell Carcinoma: Results of the GETUG-AFU 26 NIVOREN Multicenter Phase II Study. J Clin Oncol 2019;37:2008-16.

13. Goldberg SB, Gettinger SN, Mahajan A, et al. Durability of brain metastasis response and overall survival in patients with non-small cell lung cancer (NSCLC) treated with pembrolizumab. J Clin Oncol 2018;36:abstr 2009.

14. Motzer RJ, Escudier B, McDermott DF, et al. Nivolumab versus Everolimus in Advanced Renal-Cell Carcinoma. N Engl J Med 2015;373:1803-13.

15. Motzer RJ, Tannir NM, McDermott DF, et al. Nivolumab plus Ipilimumab versus Sunitinib in Advanced Renal-Cell Carcinoma. N Engl J Med 2018;378:1277-90.

16. Rini BI, Plimack ER, Stus V, et al. Pembrolizumab plus Axitinib versus Sunitinib for Advanced Renal-Cell

Cite this article as: Fallah J, Ahluwalia MS. The role of immunotherapy in the management of patients with renal cell carcinoma and brain metastases. Ann Transl Med 2019;7(Suppl 8):S313. doi: 10.21037/atm.2019.10.31
Carcinoma. N Engl J Med 2019;380:1116-27.

17. Motzer RJ, Penkov K, Haanen J, et al. Avelumab plus Axitinib versus Sunitinib for Advanced Renal-Cell Carcinoma. N Engl J Med 2019;380:1103-15.

18. Vogl UM, Bojic M, Lamm W, et al. Extracerebral metastases determine the outcome of patients with brain metastases from renal cell carcinoma. BMC Cancer 2010;10:480.

19. Juloori A, Miller JA, Parsai S, et al. Overall survival and response to radiation and targeted therapies among patients with renal cell carcinoma brain metastases. J Neurosurg 2019:1-9.

20. Theelen W, Peulen H, Lalezari F, et al. Randomized phase II study of pembrolizumab after stereotactic body radiotherapy (SBRT) versus pembrolizumab alone in patients with advanced non-small cell lung cancer: The PEMBRO-RT study. J Clin Oncol 2018;36:abstr 9023.

21. Chen L, Douglass J, Kleinberg L, et al. Concurrent Immune Checkpoint Inhibitors and Stereotactic Radiosurgery for Brain Metastases in Non-Small Cell Lung Cancer, Melanoma, and Renal Cell Carcinoma. Int J Radiat Oncol Biol Phys 2018;100:916-25. 